\title{
Case Report: A 25-Year-Old Man with Severe Non- Hemorrhagic Intraoperative Shock
}

\author{
$\underline{\text { Patrick M. Doran*, M.D., Armen Aprikian }}^{\hat{Y}}$, M.D., Joe Shuster ${ }^{\hat{Y}}$, M.D., and Laurence Green ${ }^{\hat{Y}}$, M.D. \\ * To whom correspondence should be addressed: Department of Internal Medicine, Montreal General \\ Hospital, Montreal, QC Canada H3G 2B6 \\ ${ }^{Y}$ Department of Urology, Montreal General Hospital
}

\section{THE CASE}

A 25-year-old French-Canadian man was admitted to the Montreal General Hospital urology department for elective bladder and urethral reconstruction.

\section{PAST MEDICAL HISTORY}

The patient was born with the Arnold-Chiari deformity (a congenital displacement of cerebellar tonsils, often causing obstructive hydrocephalus), spina bifida manifesta with distal sacral agenesis, and secondary urine and stool incontinence which had required him to wear protective undergarments until the age of 18 . Shortly after birth, a ventriculoperitoneal shunt had been installed, but had subsequently become nonfunctional. For his congenital bladder and bowel dysfunction, he had undergone a total of 32 operations (all performed at other institutions). These included a colostomy 13 years prior to this admission, as well as the construction of an artificial urinary sphincter 6 years later which was eventually excised because of infection. The last urologic procedure had taken place six months before this admission, and had consisted of a reconstruction of the bladder with the placement of a permanent suprapubic catheter. He had no other congenital anomalies or past surgical or medical problems of note other than numerous urinary tract infections. During the treatment of these infections, he had developed mild cutaneous allergic symptoms to cephalexin (first generation cephalosporin) and trimethoprim-sulfamethoxazole, which had required their discontinuation. He had no other known allergies and was taking no medications at the time of his admission. He denied any alcohol, tobacco, or illicit drug use.

\section{PRESENTING COMPLAINT}

The suprapubic catheter and its attached leg bag, installed six months earlier, had made his quality of life unacceptable. For this he was referred to a urologist at the Montreal General Hospital. Following consultation, the patient chose to undergo bladder reconstruction surgery, which could grant him the autonomy of a continent bladder. His course during the months preceding this admission had been otherwise devoid of any problems. A review of systems was noncontributory. 


\section{PHYSICAL EXAM}

Upon physical examination, the patient appeared well, was ambulatory, and was in no apparent distress. His vital signs were normal, with a regular heart rate of 80 , a blood pressure of $120 / 80 \mathrm{mmHg}$, a respiratory rate of 14, and a temperature of $36.2 \check{\mathrm{S}}$. The head and neck exam showed no sign of increased intracranial pressure, asymmetry, or other abnormalities. Examination of all areas showed no lymphadenopathy. The chest was normal to inspection and percussion, with normal air entry and no adventitious sounds. The precordium was unremarkable, with normal heart sounds and no murmurs. The abdomen was notable for multiple healed surgical incisions, a suprapubic catheter, and a left-sided, functional colostomy. Both of these sites appeared free of infection. The remainder of the abdominal exam was clinically normal. The integument was intact. A neurological exam was unremarkable.

\section{LABORATORY TESTS}

Routine blood work on admission showed values all within normal limits (Table 1).

An electrocardiogram showed a normal tracing with sinus rhythm at $72 / \mathrm{min}$. Dipstick urinalysis of a moderately turbid urine indicated the presence of a large amount of occult blood, a moderate amount of leukocytes and nitrites, and proteinuria in the $0.3 \mathrm{~g} / \mathrm{l}$ range.

An intravenous pyelogram performed a few months prior to admission corroborated the presence of spina bifida, and revealed normal upper urinary tracts and ureters. A cystogram done during the same period showed a bladder with a 350 cc capacity, devoid of calculi or reflux.

\section{PROCEDURES}

Shortly after his admission to the urology ward, the patient uneventfully underwent routine preparation for surgery, with enemas to clear the colon, povidone-iodine bath, and preoperative antibiotics as per protocol, with the last doses given on the day of surgery: vancomycin $500 \mathrm{mg}$ i.v. x 3 , gentamicin $80 \mathrm{mg}$ i.v. x 2 , metronidazole $750 \mathrm{mg}$ p.o. $\mathrm{x} 3$, and neomycin $1 \mathrm{~g}$ p.o. $\mathrm{x} 3$ doses. The following day, the patient was taken to the operating room for bladder reconstruction surgery. By utilizing a segment of ileum and by anastomosing one end to the reconstructed bladder neck, and the opposite end to the abdominal wall, the patient would be granted the autonomy of a readily catheterizable, continent urinary conduit.

After induction of general anesthesia and routine skin preparation and draping, a laparotomy incision was performed. The surgeon then lysed numerous adhesions in proximity of the small bowel and bladder. The bladder was then mobilized.

Approximately one hour after the first incision, the patient's hitherto stable condition suddenly deteriorated, with the appearance of acute sinus tachycardia (140-160 beats/min) and hypotension $(60 / 30 \mathrm{mmHg})$. The peritoneal cavity was immediately inspected for hemorrhage; none was found. Normal aortic pulsations were palpated. However, on a repeated occasion, the hypotension was noted to significantly worsen with each manipulation of the bowel.

The patient also developed edema of the arms, face, and upper airway. Scattered crackles were auscultated over the chest. Sequentially, he was given epinephrine $0.3 \mathrm{cc}$ 1:10,000 i.v., phenylephrine $100 \mathrm{mg}$ i.v., and dantrolene $100 \mathrm{mg}$ i.v., with no improvement. An emergency central line was uneventfully inserted in the internal jugular vein, and the patient received a 1.51 bolus of Ringer's lactate. His systolic blood pressure did not respond, remaining at $60 \mathrm{mmHg}$, and the patient was becoming increasingly cyanotic.

Diphenhydramine $50 \mathrm{mg}$ i.v. and cimetidine $300 \mathrm{mg}$ i.v. were then administered concurrently. Seconds later, 
both heart rate and blood pressure returned to near-normal values. After a $100 \mathrm{mg}$ dose of hydrocortisone was administered, a low-dose norepinephrine drip (4-8 $\mathrm{mg} / \mathrm{min})$ was started for mild recurrent hypotension (systolic 95-100 $\mathrm{mmHg}$ ). Vitals subsequently remained stable.

Once the patient had been stabilized, the surgical procedure was aborted, and the abdomen was closed. The volume of blood loss was estimated at $250 \mathrm{cc}$. Following the insertion of a nasogastric tube and a Silastic suprapubic catheter, the patient was transferred to the surgical intensive care unit. A flow-directed Swan-Ganz catheter was positioned, which showed the following readings (on norepinephrine drip $4 \mathrm{mg} / \mathrm{min}$ ) (Table 2):

Venous lactic acid measured two hours after his transfer to the unit was $2.6 \mathrm{mmol} / 1$ (Normal 0.6 - 2.4 $\mathrm{mmol} / \mathrm{l})$. Other laboratory values were noncontributory.

On the first postoperative day, the patient was alert and cooperative. He was kept intubated because of persistent oropharyngeal edema. However, his brachial and facial edema had markedly improved. He was given two more doses of i.v. hydrocortisone, which was then substituted by daily dose of oral prednisone tapered over four days. His systolic blood pressure had remained above $100 \mathrm{mmHg}$ while decreasing drip rates of norepinephrine; the latter was stopped 16 hours after it had been started.

On the second postoperative day, his oropharyngeal edema had resolved and the patient was uneventfully extubated. He continued to improve and, following extubation, he was transferred back to the urology ward.

On postoperative day 5, he was once again walking freely and eating well. On day 7, a confirmatory diagnostic procedure was performed. Upon being informed of his diagnosis, the patient remembered repeated occasions where, as a child, he had suffered from transient lip and gum swelling after blowing up balloons. To avoid similar incidents, specific precautions were adopted and duly noted in the patient's chart for future reference. The patient was discharged the following day. He returned two months later for a follow-up appointment at the urology clinic. Other than an uneventful change of his suprapubic catheter, he was completely back to normal and doing well.

The final diagnosis was latex hypersensitivity, likely having manifested itself as an anamnestic response to the intraoperative latex exposure (i.e. the surgical gloves). Previous sensitization had likely occurred during the numerous other operations and through the many years of wearing a latex-containing urinary catheter. Such a phenomenon has been previously recognized elsewhere (1-3). As a result, a latex-free Silastic catheter was deliberately used after this anaphylactic reaction. This diagnosis was initially suggested following the dramatic resolution of hypotension with the administration of a combined $\mathrm{H} 1 / \mathrm{H} 2$ antihistamine in a patient at high risk for latex hypersensitivity (i.e., with numerous exposures). Hypersensitivity to anesthetic agents (e.g., quaternary amines) was significantly less likely, due to the delay between induction and onset of symptoms. The diagnosis was finally confirmed by a strong positive skin prick test to latex antigen. This procedure had its own set of risks, since some similar individuals with spina bifida have been documented to experience anaphylaxis induced by the skin prick test itself (3). The patient's milder, localized reactions after inflating latex-containing balloons as a child may have been a harbinger of what was to come.

\section{REFERENCES}

1. Kelly KJ, Pearson ML, Kurup VP, et al. A cluster of anaphylactic reactions in children with spina bifida during general anesthesia: Epidemiologic features, risk factors, and latex hypersensitivity. Journal of Allergy and Clinical Immunology 1994;94:53-61.

2. Charous BL, Hamilton RG, Yunginger JW. Occupational latex exposure: characteristics of contact and 
systemic reactions in 47 workers. Journal of Allergy and Clinical Immunology 1994;94:12-18.

3. American Academy of Allergy and Immunology. Task Force on Allergic Reactions to Latex. Journal of Allergy and Clinical Immunology 1993;92:16-18.

\section{AUTHOR BIOGRAPHY}

Patrick M. Doran received an M.D., cM. from McGill University (Montreal, Quebec) in 1992. He is presently a third-year resident in Internal Medicine at the Montreal General Hospital (Montreal, Quebec). He will be starting an endocrinology fellowship at the Mayo Clinic (Rochester, Minnesota) next year. 